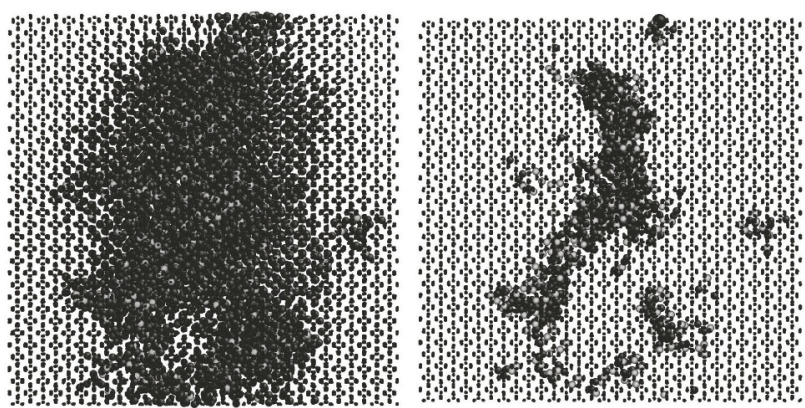

Figure 1. Radiation damage, produced by $20 \mathrm{keV}$ Th recoil in zircon at the peak of the damage (left column) and after structure relaxation (right column).

Keywords: radiation resistance of minerals, semiempirical interatomic potential method, molecular dynamics method, computer simulation of the structure and properties of the crystal, Frenkel defects

\section{MS14-P17 Nanoscale structure refinement of pyroxenes using precession electron diffraction tomography}

Damien Jacob ${ }^{1}$, Pascal Roussel ${ }^{2}$, Lukas Palatinus ${ }^{3}$, Yvan Ngassa Tankeu $^{1}$, Chiara Domeneghetti ${ }^{4}$, Fernando Camara ${ }^{5}$

1. UMET, UMR 8207 CNRS-Université Lille 1, 59655 Villeneuve d'Ascq, France

2. UCCS, UMR 8181 CNRS-Université Lille 1, Ecole Nationale Supérieure de Chimie de Lille, 59655 Villeneuve d'Ascq, France 3. Institute of Physics of the Academy of Sciences of the Czech Republic, 18221 Prague, Czech Republic

4. Dipartimento di Scienze della Terra, Università di Torino, 10125-Torino, Italy

5. Dipartimento di Scienze della Terra e dell'Ambiente, Università di Pavia, 27100-Pavia, Italy

email: damien.jacob@univ-lille1.fr

The precession electron diffraction (PED) technique [1] has been originally developed for structure determination at a submicrometer scale in a transmission electron microscope (TEM). The resulting intensities keep dynamical in nature, due to electron multiple scattering, but are more closely correlated with the structure factor values with respect to the non-precessed intensities, which is crucial for structure solution and refinement [2]. Since, many structures have been solved using PED, recently combined with the tomographic acquisition of 3D electron diffraction data (PEDT) [3]. Even more recently, a full structure refinement method based on PEDT data and dynamical calculations of diffracted intensities has been successfully proposed [4].

In the field of mineralogy, the sensitivity of PED data to structure factors can be used to refine cations fractionation on specific sites with mixed occupancies. This gives access to the ordering parameter of structures such as $\mathrm{Fe} 2+$ and $\mathrm{Mg}$ bearing pyroxenes. Ordering phenomena being time and temperature dependent, we get quantitative information concerning the thermal history and closure temperature of diffusion processes in the hosting rocks. The present study concerns the application of the full structure dynamical refinement method on natural pyroxenes from various origin, including $\left(\mathrm{Fe}^{+}, \mathrm{Mg}\right) \mathrm{SiO}_{3}$ orthopyroxene (already studied thanks to a 2D zone-axis approach [5]) and $\left(\mathrm{Fe} 2^{+}, \mathrm{Mg}, \mathrm{Ca}\right) \mathrm{SiO}_{3}$ clinopyroxene. The refinement of 3D electron diffraction data against dynamical calculations enables the estimation of the (Fe, $\mathrm{Mg}$ ) occupancies on M1 and M2 sites of the structures with a good precision, the refined values being closed to those deduced from XRD experiments at the grain scale. We will discuss on the accuracy and sensitivity of the method and on its potential application in the field of geothermometry using local analysis of terrestrial or extra-terrestrial pyroxene bearing rocks.

[1] Vincent, R., and Midgley, P.A.

(1994) Ultramicroscopy, 53(3), 271-282.

[2] Sinkler, W., and Marks, L.D. (2010) Zeitschrift Fur Kristallographie, 225(2-3), 47-55. 
[3] Mugnaioli, E., Gorelik, T. E. \& Kolb, U. (2009). Ultramicroscopy 109, 758-765

[4] Palatinus, L., Petricek, V., and Correa, C.A. (2015). Acta Crystallographica Section A, 71(2), 1-10.

[5] Jacob, D., Palatinus, L., Cuvillier, P., Leroux, H., Domeneghetti, C., and Camara, F. (2013) American Mineralogist, 98(8-9), 1526-1534.

Keywords: electron crystallography, structure refinement, pyroxene, cooling history

\section{MS14-P18 Structural distortion of biogenic aragonite in Ranella Olerea mollusc shell layers}

Salim Ouhenia ${ }^{1}$, Daniel Chateigner ${ }^{2}$, Stéphanie Gascoin ${ }^{2}$

1. Equipe de cristallographie et de simulation des matériaux, Laboratoire de physique des matériaux et catalyse, Université de Bejaia, Bejaia 06000, Algérie.

2. Laboratoire CRISMAT-ENSICAEN (CNRS UMR 6508), Université de Caen Basse-Normandie, 6 bd M. Juin, 14050 Caen, France.

email: ouhenia@yahoo.fr

Mollusc shells, mainly made of calcite and aragonite crystalline polymorphs of calcium carbonate, are fascinating organic-mineral biocomposites with high mechanical performances, as they attain a fascinating increase in both strength and toughness compared to the geological mineral. The major part of organic materials is intercrystalline, and in a minor way intracrystalline (Pokroy et al., 2006).The organic represents less than 5\% in volume, is a biopolymer dispersed in inorganic crystal of calcium carbonate (Barthelat and Espinosa, 2007). This organic part behaves as nanometer growth-control of the inorganic crystals and also plays an important role in stopping crack propagation in nacre (Cortie et al.,2006). This has stimulated chemists and materials scientists to design and synthesize high performance materials with a microstructure similar to that of nacre (Wang et al., 2013). In the present work we made use of Combined Analysis to determine the structure and unit-cell distortions of constituting aragonite crystallites of the shell layers (figure 1) of the gastropod Ranella olearea. This approach was chosen because it allows working on real samples, without grinding operation (Ouhenia et al., 2008). SEM analyses show the presence of three distinct layers; an inner layer composed of Radial Lamellar, an intermediate comarginal crossed lamellar layer and an outer crossed lamellar layer. The refinement of X-ray diffraction diagrams, gives quantitatively the structure of the three layers and their respective aragonite unit-cell distortions. An anisotropic unit-cell distortion is quantified for the three layers which is attributed to the combined effects of inter- and intra-crystalline macromolecules.

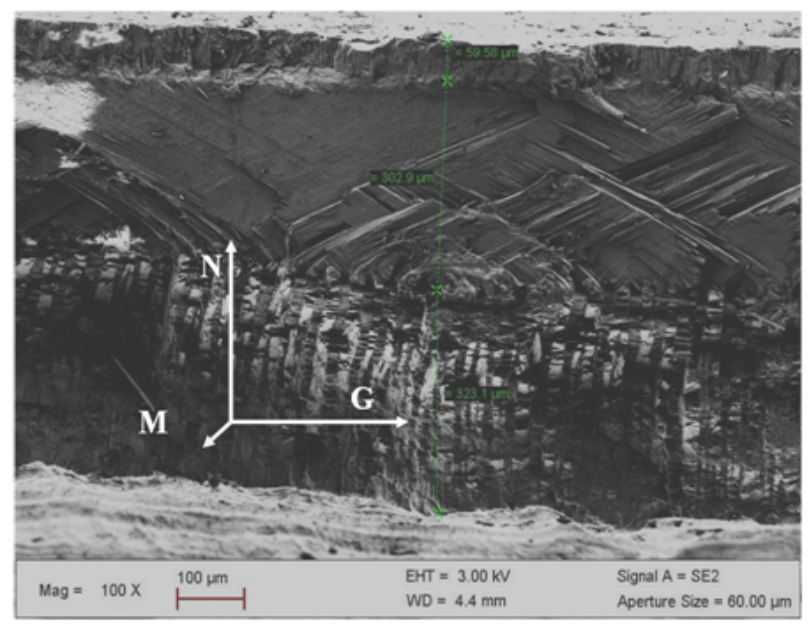

Figure 1. figure 1: Cross-section SEM image of the fractured shell at the location indicated in $\mathrm{G}, \mathrm{M}$ and $\mathrm{N}$ indicate the Growth, Margin and Normal directions, respectively. 